\title{
Expression of the endogenous and heterologous clavulanic acid cluster in Streptomyces flavogriseus: why a silent cluster is sleeping
}

\author{
R. Álvarez-Álvarez • Y. Martínez-Burgo • R. Pérez-Redondo • \\ A.F. Braña • J.F. Martín • P. Liras
}

Received: 18 June 2013 /Revised: 22 July 2013 / Accepted: 24 July 2013 / Published online: 22 August 2013

(C) Springer-Verlag Berlin Heidelberg 2013

\begin{abstract}
Clusters for clavulanic acid (CA) biosynthesis are present in the actinomycetes Streptomyces flavogriseus ATCC 33331 and Saccharomonospora viridis DSM 43017. These clusters, which are silent, contain blocks of conserved genes in the same order as those of the Streptomyces clavuligerus CA cluster but assembled in a different organization. S. flavogriseus was grown in nine different media, but clavulanic acid production was undetectable using bioassays or by high-performance liquid chromatography analyses. Reverse-transcriptase polymerase chain reaction (RT-PCR) of S. flavogriseus CA biosynthesis genes showed that the regulatory genes $c c a R$ and $c l a R$ and some biosynthetic genes were expressed whereas expression of cyp, $\operatorname{orf12}$ orf13, and oppA2 was undetectable. The $c c a R$ gene of $S$. clavuligerus was unable to switch on CA production in $S$. flavogriseus:: $\left[\mathrm{P}_{\text {fur }}-c c a R_{\mathrm{C}}\right]$, but insertion of a cosmid carrying
\end{abstract}

Electronic supplementary material The online version of this article (doi:10.1007/s00253-013-5148-7) contains supplementary material, which is available to authorized users.

R. Álvarez-Álvarez · Y. Martínez-Burgo · J. Martín · P. Liras $(\bowtie)$ Microbiology Section, Department of Molecular Biology, Faculty of Biology and Environmental Sciences, Vegazana Campus,

University of León, León 24071 Spain

e-mail: paloma.liras@unileon.es

R. Álvarez-Álvarez

e-mail: ralva@unileon.es

Y. Martínez-Burgo

e-mail:ymarb@unileon.es

R. Álvarez-Álvarez $\cdot$ R. Pérez-Redondo $\cdot$ P. Liras Institute of Biotechnology INBIOTEC, Av. Real 1, León 24006 Spain

R. Pérez-Redondo

e-mail: r.perez.redondo@unileon.es

\section{A. Braña}

Fundamental Biology Department, Faculty of Medicine and University Institute of Oncology from the Principado de Asturias (I.U.O.P.A), University of Oviedo, Oviedo 33006 Spain e-mail: afb@uniovi.es the $S$. clavuligerus CA cluster (not including the ccaR gene) conferred clavulanic acid production on $S$. flavogriseus::[SCos$\mathrm{CA}$ ] particularly in TBO and YEME media; these results suggests that some of the $S$. flavogriseus $\mathrm{CA}$ genes are inactive. The known heptameric sequences recognized by $\mathrm{CcaR}$ in $S$. clavuligerus are poorly or not conserved in S. flavogriseus. Quantitative RT-PCR analysis of the CA gene clusters of $S$. clavuligerus and S. flavogriseus showed that the average expression value of the expressed genes in the former strain was in the order of 1.68-fold higher than in the later. The absence of CA production by $S$. flavogriseus can be traced to the lack of expression of the essential genes cyp, orf12, orf13, orf14, and oppA2. Heterologous expression of $S$. clavuligerus CA gene cluster in S. flavogriseus::[SCos-CA] was 11- to 14-fold lower than in the parental strain, suggesting that the genetic background of the host strain is important for optimal production of CA in Streptomyces.

Keywords Genomes · Clavulanic acid · Gene clusters · Heterologous expression · Streptomyces clavuligerus. Streptomyces flavogriseus $\cdot$ Saccharomonospora viridis

\section{Introduction}

Clavulanic acid (CA) is a $\beta$-lactamase inhibitor with clavam structure produced industrially from cultures of Streptomyces clavuligerus. The CA molecule is formed by condensation of a five-carbon fragment of arginine (the ornithine moiety) with a three-carbon glyceraldehyde-3-phosphate unit ( Khaleeli et al. 1999; Valentine et al. 1993) by the carboxyethylarginine (CEA) synthase encoded by ceaS2 (Pérez-Redondo et al. 1999). The product of this condensation, carboxyethylarginine, is cyclized to proclavaminic acid by the $\beta$-lactam synthetase (bls2) (Bachmann et al. 1998) and modified in sequential steps by the clavaminate synthetase (cas2) and the proclavamic acid hydrolase (pah2) that removes the amidino group of arginine 
to produce clavaminic acid (Marsh et al. 1992; Aidoo et al. 1994) (Fig. 1a). The known late steps of the CA pathway involve at least two enzymes, the glycyl clavaminic acid synthase (encoded by gcaS) forming the peptide-like intermediate $\mathrm{N}$-glycyl-clavaminic acid. This compound is the precursor of clavaldehyde from which clavulanic acid is formed by the final enzyme of the pathway, clavaldehyde reductase (Car) (Pérez-Redondo et al. 1998; Nicholson et al. 1994).

Besides the biosynthetic genes encoding enzymes of the pathway, some additional genes located in the CA cluster are important for CA production. These include oppAl and oppA2, encoding two oligopeptide permeases; cyp, encoding a P450 cytochrome monooxygenase; and orf 12 , encoding a putative lipoprotein. Disruption of each of these genes led to lack of CA production (Bachmann et al. 1998; Mellado et al. 2002; Lorenzana et al. 2004). Other genes in the cluster (orf13, orf14, and oat 2) are required for maximum CA production, but their disruption still allows some production of the $\beta$-lactamase inhibitor (for a review, see Liras et al. 2011).
The CA gene cluster of $S$. clavuligerus is adjacent to the cephamycin $\mathrm{C}$ gene cluster (including 17 genes from bla to $p b p R)$. A SARP-type regulatory gene, $c c a R$, controlling clavulanic acid and cephamycin $\mathrm{C}$ biosynthesis (PérezLlarena et al. 1997a) is located within the cephamycin C gene cluster. A second regulatory gene, claR, of the LysR family, located in the CA cluster, controls the expression of genes for the late steps of the CA pathway (Pérez-Redondo et al. 1998; Paradkar et al.1998).

The ability to produce $\beta$-lactams and other secondary metabolites is usually restricted to a few species within different genera (Martín et al. 2000; Martín and Liras 2010), and this ability is due to (1) the ancestral assembling of the gene cluster or (2) to the acquisition of genes by horizontal transfer either in the same species (there are two sets of paralogous clavam genes in S. clavuligerus) or between different species (Liras et al. 1998). The availability of microbial genomes is revealing the existence of gene clusters for secondary metabolites which are not expressed. The awakening of these silent clusters is a

a

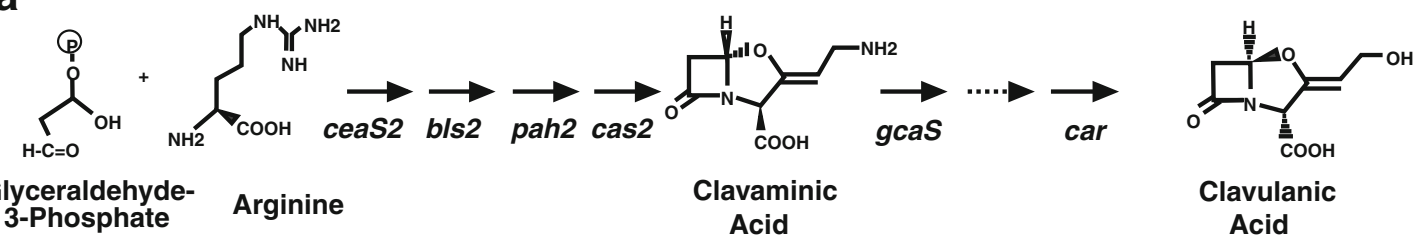

b
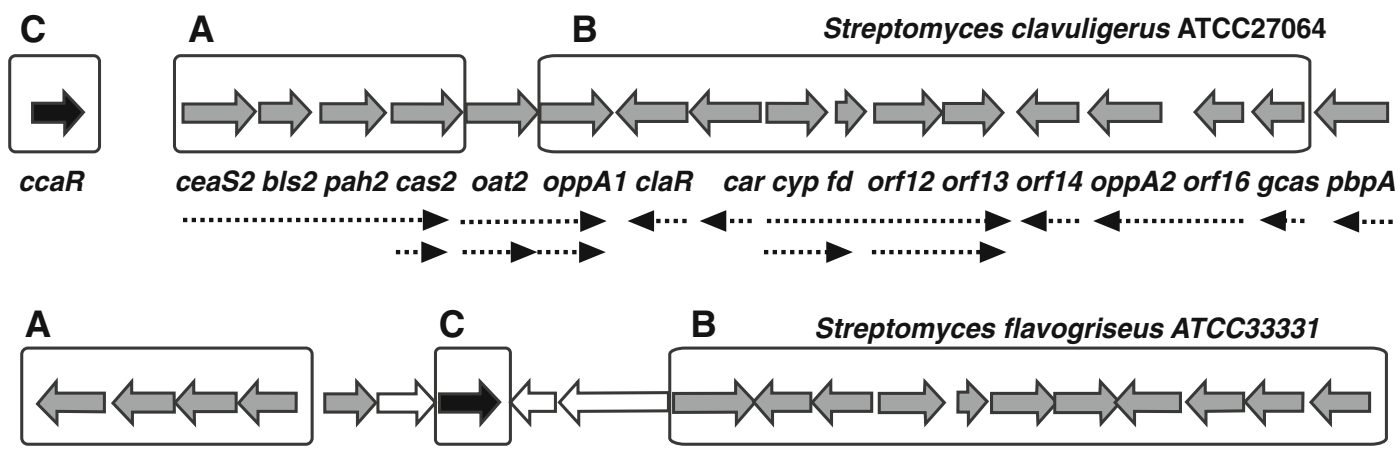

cas2 pah2 bls2 ceaS2 oat2 ccaR oppA1 claR car cyp fd orf12 orf13 orf14 oppA2 orf16 gcas

Sf-0553 Sf-0551 Sf-0550

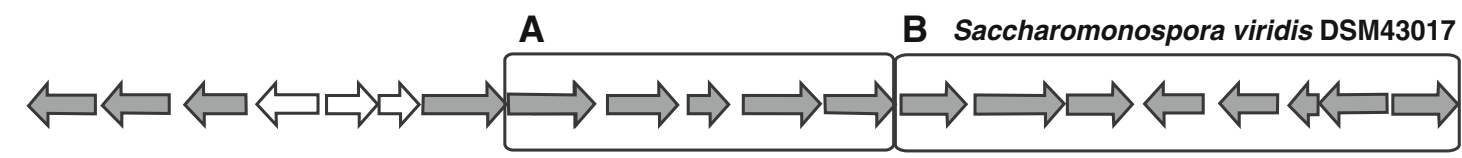

Sv-33300 oat2 claR Sv-33330 oppA1 ceaS2 bls2 gcas pah2 cas2 orf16 opA2 orf14 orf13 orf12 fd cyp car Sv-33340 Sv-33350

Fig. 1 Clavulanic acid biosynthesis pathway and gene clusters. a Scheme of the clavulanic acid pathway. The gene encoding enzymes involved in the different steps are indicated. b Clavulanic acid gene cluster in $S$. clavuligerus, S. flavogriseus, and Sac. viridis. The transcriptional pattern of the $S$. clavuligerus cluster is indicated with broken arrows. The grouped subclusters (blocks A, B, and C) referred in the text are enclosed in square boxes. The regulatory gene ccaR (block $\mathrm{C}$ ) is indicated with a black-filled arrow 
challenge for the pharmaceutical industry and requires understanding of the molecular basis for this lack of expression (Laureti et al. 2011).

Taking into account the increasing number of fully or partially sequenced microbial genomes (www.ncbi.nlm.nih. gov), we undertook the search for other putative CA gene clusters in different microorganisms that may contain (or lack) additional genes. Our aim was to test production of CA or related compounds in different media by highperformance liquid chromatography (HPLC) and biological assays to determine if those novel CA gene clusters were expressed.

In this work, the CA cluster of Streptomyces flavogriseus and Saccharomonospora viridis (hereafter abbreviated as Sac. viridis) was analyzed, and the production of CA by these strains was tested. In addition, the heterologous expression of the $S$. clavuligerus CA cluster in $S$. flavogriseus was allowed to identify nonexpressed genes that explain the silent nature of the $S$. flavogriseus CA cluster.

\section{Materials and methods}

\section{Strains and culture conditions}

Streptomyces strains used in this work are described in Table 1 . They were grown at $28^{\circ} \mathrm{C}$ and $220 \mathrm{rpm}$ in $500-\mathrm{ml}$ baffled flasks containing $100 \mathrm{ml}$ TSB medium for $24 \mathrm{~h}$ to an $\mathrm{OD}_{600 \mathrm{~nm}}$ of 6.5 and used to inoculate $(5 \% v / v)$ the final cultures. The following media were used to grow the Streptomyces species: defined SA and MG as described by
Paradkar et al. (1998) and Doull and Vining (1989). Complex TSB, YEME, R5 media, MEY, or MS as described by Kieser et al. (2000), TBO as described by Higgens et al. (1974), and ISP4 (Difco $\left.{ }^{\mathrm{TM}}\right)$. Kanamycin $(50 \mu \mathrm{g} / \mathrm{ml})$, thiostrepton $(10 \mu \mathrm{g} /$ $\mathrm{ml})$, hygromycin $(50 \mu \mathrm{g} / \mathrm{ml})$, and nalidixic acid $(25 \mu \mathrm{g} / \mathrm{ml})$ were added to the cultures when required.

\section{Clavulanic acid analysis}

Clavulanic acid production was quantified using bioassay with Klebsiella pneumoniae ATCC 29665 as test strain as indicated by Romero et al. (1984). When required, the bioassays were repeated in plates lacking penicillin G. Broth of the cultures of $S$. flavogriseus::[SCos-AC] was derivatized with imidazole as described by Foulstone and Reading (1982). These samples were analyzed by HPLC in a SunFire column $(5 \mu \mathrm{m}, 4.6 \times 250 \mu \mathrm{mm}$; Waters, Milford, MA, USA), using an isocratic elution with acetonitrile and $0.1 \%$ trifluoroacetic acid in water $(10: 90)$ at $1 \mathrm{ml} / \mathrm{min}$. Peaks were detected at $318 \mathrm{~nm}$. For HPLC-MS analysis, an Alliance chromatographic system coupled to a ZQ4000 mass spectrometer and an Atlantis T3 column $(3 \mu \mathrm{m}, 2.1 \times 150 \mathrm{~mm}$; Waters, Milford) was used. Samples $(10 \mu \mathrm{l})$ were injected and eluted with $0.1 \%$ formic acid in water during $4 \mathrm{~min}$, followed by a linear gradient from 0 to $40 \%$ acetonitrile over $16 \mathrm{~min}$ at $0.2 \mathrm{ml} / \mathrm{min}$. MS analysis were done by electrospray ionization in the negative mode, with a capillary voltage of $3 \mathrm{kV}$ and a cone voltage of $20 \mathrm{~V}$. Clavulanic acid was detected by selected ion recording at $m / z$ 198. Pure clavulanic acid (Antibióticos SA, León, Spain) was used as standard.

Table 1 Streptomyces strains used in this work

\begin{tabular}{|c|c|c|}
\hline Strain & Origin & Characteristics of the strain \\
\hline S. clavuligerus ATCC 27064 & ATCC & Wild-type strain \\
\hline S. clavuligerus $\Delta c c a R:: t s r$ & Alexander and Jensen 1998 & Clavulanic acid and cephamycin $\mathrm{C}$ non producer. Thiostrepton resistant \\
\hline $\begin{array}{l}\text { S. clavuligerus }(\Delta c c a R:: t s r):: \\
\quad\left[\mathrm{P}_{\text {fur }}-c c a R_{\mathrm{F}}\right]\end{array}$ & This work & $\begin{array}{l}\text { Clavulanic acid and cephamycin } \mathrm{C} \text { non producer. Thiostrepton resistant. } \\
\text { It carries, integrated in the genome, the } c c a R_{\mathrm{F}} \text { gene expressed from } \\
\text { the } \mathrm{P}_{\text {fur }} \text { promoter }\end{array}$ \\
\hline S. flavogriseus ATCC 33331 & ATCC & Wild-type strain \\
\hline S. flavogriseus:: $\left[\mathrm{P}_{\text {fur }}-c c a R_{\mathrm{C}}\right]$ & This work & $\begin{array}{l}\text { Clavulanic acid non producer. It carries the } c c a R_{C} \text { gene expressed from } \\
\text { the } \mathrm{P}_{\text {fur }} \text { promoter of } S \text {. coelicolor, integrated in the genome. Hygromycin } \\
\text { B resistant }\end{array}$ \\
\hline S. flavogriseus::[SCos-CA] & This work & $\begin{array}{l}\text { Clavulanic acid producer. It carries the [SCos-AC] containing the clavulanic } \\
\text { acid gene cluster of } S \text {. clavuligerus integrated in the genome. Kanamycin } \\
\text { and tetracycline resistant }\end{array}$ \\
\hline $\begin{array}{l}\text { S. flavogriseus::[}\left[\mathrm{P}_{\text {fur }}-c c a R_{\mathrm{C}}\right]:: \\
\quad[\text { SCos-CA }]\end{array}$ & This work & $\begin{array}{l}\text { Clavulanic acid producer. It carries integrated in the genome [SCos-AC] } \\
\text { and the } c c a R_{\mathrm{C}} \text { gene expressed from the } \mathrm{P}_{\text {fur }} \text { promoter. Kanamycin, } \\
\text { tetracycline, and hygromycin B resistant }\end{array}$ \\
\hline S. coelicolor M1146 & Gomez-Escribano and Bibb 2011 & Strain for expression of heterologous gene clusters \\
\hline
\end{tabular}


Nucleic acid manipulation

\section{Oligonucleotides}

Oligonucleotides used in this work (Laboratorios Conda, Madrid, Spain) are shown in Table S1. The oligonucleotides designed to discriminate orthologous genes of S. clavuligerus ATCC 27064 and S. flavogriseus ATCC 33331 were tested in both strains to ensure the specific amplification of the analyzed gene.

\section{Isolation of nucleic acid}

DNA was obtained by the modified Kirby method as described by Kieser et al. (2000). RNA was obtained using the RNeasy kit (Qiagen). Samples to extract RNA were taken at 24 and $48 \mathrm{~h}$ of culture. The RNA was quantified in a NanoDrop spectrophotometer, the integrity analyzed using a Bioanalyzer 2100 (Agilent Technologies), and the chips included in the RNA 6000 Nano LabChip ${ }^{\circledR}$ kit (Agilent).

\section{Construction of plasmids $\left[P_{f u r}-c c a R_{F}\right]$ and $\left[P_{f u r}-c c a R_{C}\right]$}

Using the DNA of $S$. flavogriseus and the oligonucleotides Sfla_0552-D and Sfla_0552-R (Table S1), an 854-bp DNA fragment was amplified containing NcoI and HindIII digestion sizes at the ends. This DNA fragment, containing the $c c a R_{\mathrm{F}}$ gene (Sfla_0552 gene, orthologous to $c c a R_{\mathrm{C}}$ ) was ligated to plasmid pUFurReg digested with the same enzymes to introduce $c c a R_{\mathrm{F}}$ in phase downstream of the constitutive promoter $\mathrm{P}_{\text {fur }}$ (Ortiz de Orue Lucana et al. 2003) to avoid the regulation by sigma-antisigma factors of $c c a R$ (Bignell et al. 2005). The $c c a R_{\mathrm{F}}$-containing fragment was isolated with BamHI-HindIII, filled with Klenow and subcloned in the EcoRV site of the integrative vector pMS82 (Matthew et al. 2003). The resulting plasmid $\left[\mathrm{P}_{\text {fur }}-c c a R_{\mathrm{F}}\right]$ was transformed in E. coli ET12567[pUZ8002], which was later conjugated with S. clavuligerus $\Delta c c a R:: t s r$ to give S. clavuligerus $(\Delta c c a R:: t s r)::\left[\mathrm{P}_{\text {fur }}-c c a R_{\mathrm{F}}\right]$. To construct $\left[\mathrm{P}_{\text {fur }}-\mathrm{ccaR}_{\mathrm{C}}\right]$, the $S$. clavuligerus $c c a R_{\mathrm{C}}$ gene was amplified by PCR using oligonucleoties SCLAV_ccaR-D and SCLAV_ccaR-R which carry at the ends an NcoI and a HindIII site, respectively. The following steps were carried out in parallel with those described for $\left[\mathrm{P}_{\text {fur }}-c c a R_{\mathrm{F}}\right]$.

\section{Location and analysis of the [SCos-CA] cosmid}

Two DNA fragments from the clavulanic acid gene cluster were amplified. They corresponded to (1) a 502-bp DNA fragment internal to ceaS2 which was amplified with oligonucleotides ceaS2_D and ceaS2_R and (2) a 516-bp DNA fragment containing the intergenic region and part of the orfl8 and gcaS genes, which was amplified with oligonucleotides orf18gcaS_D and orf18-gcaS_R. The amplified regions were used to scan a SuperCos gene library of $S$. clavuligerus DNA (RoblesReglero and Liras, unpublished results). One cosmid giving positive hybridization with both probes, analyzed by restriction digestion, PCR of internal zones, and partial sequencing, was found to carry the whole CA gene cluster. This cosmid, which will be named [SCos-CA], carries an insert including genes from ceaS2 to gcaS but not carry the $c c a R$ gene.

Vector pFL1272, a pIJ787-derived plasmid (Eustáquio et al. 2005), was digested with XbaI, and the integration cassette (containing the int and tet ${ }^{\mathrm{R}}$ genes, and the att site of $\phi C 31)$ was isolated and filled with Klenow in the presence of thymines and cytosines. Cosmid [SCos-CA] was digested at a single site with HindIII, the ends were filled with Klenow in the presence of adenines and guanines, and the linear fragment was ligated to the pFL1272 integration cassette. The resulting construction was transformed in E. coli $\mathrm{DH} 5 \alpha$ and colonies resistant to both kanamycin and tetracycline were isolated. After the confirmation of the correct construction, the cosmid was introduced in E. coli ET12567[pUZ8002] which was conjugated with spores of S. flavogriseus. Exconjugants resistant to kanamycin were tested by PCR for the presence of the [SCos-CA] cosmid. Ninety percent of the exconjugants tested showed amplification by PCR of a 1,700-bp DNA fragment containing oppA2 which was not amplified when DNA from S. flavogriseus ATCC 33331 was used.

\section{$R T-P C R$}

The one-step reverse-transcriptase polymerase chain reaction (RT-PCR) mixture contained in a $20-\mu l$ volume RNA template (200 ng), reaction mixture $1 \mathrm{X}$, oligonucleotides $0.5 \mathrm{mM}$ each, DMSO (5\%), SuperScriptTM II reverse transcriptase, and Platinum $^{\circledR}$ Taq 2 U (Invitrogen, Carlsbad, CA, USA). Control reactions contained Platinum ${ }^{\circledR}$ Taq DNA polymerase but no reverse transcriptase. The reaction to synthesize cDNA was carried out at $55^{\circ} \mathrm{C}$ for $30 \mathrm{~min}$. Amplification by PCR of the cDNA ( 30 or 35 cycles) was as follows: $95^{\circ} \mathrm{C} / 30 \mathrm{~s} ; 60^{\circ} \mathrm{C} /$ $30 \mathrm{~s} ; 72{ }^{\circ} \mathrm{C} / 40 \mathrm{~s}$, and a final extension of $72{ }^{\circ} \mathrm{C} / 10 \mathrm{~min}$. Controls (not shown) to test the $S$. clavuligerus CA gene expression in the different media were always included using oligonucleotides already tested for $S$. clavuligerus CA gene amplification (Santamarta et al. 2011; López-García et al. 2010).

\section{$R T-q P C R$}

Gene expression analysis by reverse-transcriptase quantitative polymerase chain reaction (RT-qPCR) was performed as previously described (Kurt et al. 2013). The quantification of gene expression was performed by the $2^{-\Delta \mathrm{Ct}}$ method (Livak and Schmittgen 2001; Schmittgen and Zakrajsek 2000), using the gene $h r d B$ as internal control (Aigle et al. 2000). 


\section{Results}

Genes for clavulanic acid biosynthesis occur in other actinomycetes different from S. clavuligerus

The complete clavulanic acid gene cluster occurs infrequently in the genome of actinomycetes. This cluster is located side by side to the cephamycin C gene cluster in S. clavuligerus, Streptomyces jumonjinensis, and Streptomyces katsuharamanus (Ward and Hodgson 1993), but no information on the CA gene cluster sequence of the last two species is available. Streptomyces antibioticus Tü1718, a clavam producer (Nobary and Jensen 2012; Janc et al. 1995), contains a complete ceaSbls-pah-cas cluster for the early genes of CA biosynthesis but lacks the genes for the late steps of the CA pathway. A complete CA cluster is present in S. flavogriseus ATCC 33331 (Sfla 0539 to Sfla_0558) (Fig. 1B), previously unknown to be a clavulanic acid producer, and in Sac. viridis DSM 43017 (Svir_33300 to Svir 33490); this is an actinomycete, member of the Pseudonocardiaceae family, with Gram-negative staining properties (Pati et al. 2009) and a circular chromosome different to Streptomyces species which have a linear chromosome. In addition, some genes of the CA cluster are present in the Grampositive bacterium Anoxybacillus flavithermus WK1.

The clusters in S. flavogriseus ATCC 33331 and Sac. viridis DSM 43017 contain all the CA genes and show a strong conservation of several blocks of genes of the known CA cluster but with important differences between them. All the CA proteins of S. flavogriseus show a similar length and higher percentage of amino acid identity with those of $S$. clavuligerus (ranging from $88 \%$ for OppA1 to $61 \%$ for $\mathrm{ClaR}$ ) than with those of Sac. viridis (Table 2). In Sac. viridis, the claR gene, encoding the LysR-type regulator of the late steps of the pathway, has a poor identity (31\%) with respect to $S$. clavuligerus claR (as compared to the $61 \%$ of S. flavogriseus claR), but the identity is spread throughout the whole gene what excludes frameshift mutations.

The GcaS protein encoded using S. flavogriseus and Sac. viridis supports the annotation of a short version for $S$. clavuligerus GcaS as suggested by Arulanantham et al. (2006). Also, the putative membrane protein encoded by orf13 of $S$. clavuligerus has a stretch of 26 amino acids at the C-terminal end (amino acids 433 to 458) which are not present in the other orthologous proteins. However, the orf12-encoded putative lipoprotein of $S$. clavuligerus contains several internal amino acid stretches (amino acids 1-18, 49-55, 75-80, 146$155,198-226,346-358,380-388$, and 433-458) that are not present in the S. flavogriseus and Sac. viridis orthologous.

Organization of the clavulanic acid gene cluster in S. flavogriseus and Sac. viridis

All the genes described in the CA cluster of $S$. clavuligerus are present in S. flavogriseus with the exception of $p b p A$. This gene encodes a penicillin $\mathrm{G}$ binding protein (Ishida et al. 2006) but its involvement in CA biosynthesis is not clear (Liras et al. 2011). Genes with 79 and $39 \%$ identity to the orthologous S. clavuligerus pbpA are $3.25 \mathrm{Mb}$ and $3.2 \mathrm{~kb}$ away, respectively, from the CA cluster in S. flavogriseus and Sac. viridis.

Three blocks of genes (Fig. 1b) are almost identical in the CA cluster of the three strains and in the clavam producer $S$. antibioticus:

\section{Block $A$}

Block A (Fig. 1b) includes the genes for the early steps of CA biosynthesis (ceaS2-bls2-pah2-cas2) which are cotranscribed in S. clavuligerus (Santamarta et al. 2011), next to oat2, a non-essential gene related to arginine utilization, which is in the opposite orientation in S. flavogriseus and Sac. viridis. These genes are in the same order in S. clavuligerus, $S$. flavogriseus, and S. antibioticus (Nobary and Jensen 2012), but genes in block A are in the opposite orientation with respect to block B in S. flavogriseus (Fig. 1b).

\section{Block B}

A large set of genes (block B in Fig. 1b) in S. clavuligerus carries oppA1-claR-car-cyp-fd-orf12-orf13-orf14-oppA2-orf16gcaS and includes late biosynthetic, regulatory, and signal transport genes. This block is fully conserved in S. flavogriseus and has the opposite orientation in relation to block A in $S$. flavogriseus and Sac. viridis as compared to S. clavuligerus. It is interesting that in Sac. viridis, block B lacks the claR, oppAl, and gcaS genes, which are located elsewhere in the cluster. In Sac. viridis, block A contains in addition, inserted between bls 2 and pah2, the gcaS gene which in S. clavuligerus and S. flavogriseus is located in block B.

The regulatory gene ccaR of $S$. flavogriseus is located inside the CA cluster at a different location than in $S$. clavuligerus

\section{Block $C$}

The third block (Fig. 1b) includes only the regulatory gene $c c a R$. This gene encodes the SARP-type regulatory protein CcaR that, in $S$. clavuligerus, binds heptameric sequences upstream of ceaS2 and claR and activates expression of these genes and of those located downstream of ceaS2 (Santamarta et al. 2011). The ccaR gene in S. clavuligerus is located at $21.5 \mathrm{~kb}$ away from the CA cluster, central to the cephamycin C gene cluster (Pérez-Llarena et al. 1997a). S. flavogriseus lacks a cephamycin $\mathrm{C}$ biosynthesis cluster, and in this species, ccaR is located downstream of Sfla_0553, in the middle of the CA cluster. 
Table 2 Comparative analysis of clavulanic acid biosynthesis genes in S. clavuligerus ATCC 27064, S. flavogriseus ATCC 33331, and Sac. viridis DSM 43017

\begin{tabular}{|c|c|c|c|c|c|c|c|c|c|}
\hline $\begin{array}{l}\text { S. clavuligerus } \\
\text { ATCC } 27064\end{array}$ & Gene & Product & $\begin{array}{l}\text { Size } \\
(\mathrm{aa})^{\mathrm{a}}\end{array}$ & $\begin{array}{l}\text { S. flavogriseus } \\
\text { ATCC } 33331\end{array}$ & $\begin{array}{l}\text { Size } \\
(\mathrm{aa})^{\mathrm{a}}\end{array}$ & $\begin{array}{l}\text { Identity/ } \\
\text { similarity }(\%)^{\mathrm{b}}\end{array}$ & $\begin{array}{l}\text { Sac. viridis } \\
\text { DSM } 43017\end{array}$ & $\begin{array}{l}\text { Size } \\
(\mathrm{aa})^{\mathrm{a}}\end{array}$ & $\begin{array}{l}\text { Identity/ } \\
\text { similarity }(\%)^{\mathrm{b}}\end{array}$ \\
\hline SCLAV_4197 & ceas2 & Carboxyethylarginine synthase 2 & 586 & Sfla_0555 & 571 & $84 / 90$ & Svir_33370 & 556 & $68 / 79$ \\
\hline SCLAV_4196 & $b l s 2$ & CEA beta-lactam-synthase 2 & 513 & Sfla_0556 & 509 & $67 / 76$ & Svir_33380 & 512 & $51 / 63$ \\
\hline SCLAV_4195 & pah2 & Proclavaminate amidinohydrolase 2 & 313 & Sfla_0557 & 316 & $84 / 92$ & Svir_33400 & 343 & $68 / 78$ \\
\hline SCLAV_4194 & cas2 & Clavaminate synthase 2 & 325 & Sfla_0558 & 324 & $78 / 85$ & Svir_33410 & 323 & $63 / 75$ \\
\hline SCLAV_4193 & oat2 & Glutamate N-acetyltransferase 2 & 399 & Sfla_0554 & 392 & $80 / 86$ & Svir_33310 & 385 & $70 / 79$ \\
\hline SCLAV_4192 & oppA1 & ABC-type dipeptide transport system & 564 & Sfla_0549 & 553 & $88 / 92$ & Svir_33360 & 549 & $83 / 90$ \\
\hline SCLAV_4191 & claR & Transcriptional activator & 432 & Sfla_0548 & 488 & $61 / 73$ & Svir_33320 & 468 & $31 / 48$ \\
\hline SCLAV_4190 & car & Clavaldehyde reductase & 248 & Sfla_0547 & 247 & $67 / 80$ & Svir_33490 & 247 & $68 / 79$ \\
\hline SCLAV_4189 & cyp & Cytochrome P450-SU2 & 408 & Sfla_0546 & 410 & $77 / 87$ & Svir_33380 & 406 & $71 / 83$ \\
\hline SCLAV_4188 & $f d$ & Ferredoxin & 71 & Sfla_0545 & 70 & $69 / 80$ & Svir_33470 & 68 & $60 / 69$ \\
\hline SCLAV_4187 & orf12 & Beta-lactamase-like protein & 458 & Sfla_0544 & 432 & $61 / 67$ & Svir_33460 & 424 & $52 / 66$ \\
\hline SCLAV_4186 & orf13 & Integral membrane protein & 340 & Sfla_0543 & 327 & $62 / 73$ & Svir_33450 & 314 & $63 / 75$ \\
\hline SCLAV_4185 & orf14 & Acetyltransferase GNAT family protein & 339 & Sfla_0542 & 343 & $62 / 72$ & Svir_33440 & 331 & $62 / 72$ \\
\hline SCLAV_4183 & оррA2 & ABC-type dipeptide transport system & 562 & Sfla_0541 & 567 & $81 / 88$ & Svir_33430 & 562 & $76 / 84$ \\
\hline SCLAV_4182 & $\operatorname{orf16}$ & DUF482 domain-containing protein & 401 & Sfla_0540 & 391 & $70 / 80$ & Svir_33420 & 384 & $68 / 78$ \\
\hline SCLAV_4181 & gcas & Biotin carboxylase & 529 & Sfla_0539 & 429 & $85 / 89$ & Svir_33390 & 428 & $76 / 85$ \\
\hline SCLAV_4180 & pbpA & Penicillin-binding protein & 529 & Sfla_3620 & 494 & $79 / 89$ & Svir_00380 & 488 & $39 / 56$ \\
\hline SCLAV_4204 & $c c a R$ & SARP-type regulator & 262 & Sfla_0552 & 262 & $45 / 63$ & - & - & - \\
\hline SCLAV_4202 & $b l p$ & $\beta$-lactamase inhibitory protein & 182 & Sfla_0521 & 186 & $44 / 56$ & - & - & - \\
\hline
\end{tabular}

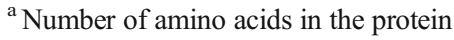

${ }^{\mathrm{b}}$ Amino acid identity/similarity to the orthologous $S$. clavuligerus protein

Surprisingly, no $c c a R$ gene is present in the CA cluster of Sac. viridis; the gene with highest similarity to $c c a R$ is Svir_28940 (32\% amino acid identity), located about $40 \mathrm{~kb}$ away from the CA cluster.

Heterogeneous genes inserted in the CA cluster of S. flavogriseus and Sac. viridis

Three genes, unrelated to the $S$. clavuligerus CA gene cluster, are inserted into the CA cluster of S. flavogriseus. One gene, Sfla_0550, encodes a protein with $53 \%$ identity to NocE, a protein with a SGNH hydrolase-type esterase domain encoded by SCLAV_5162 in S. clavuligerus but outside the CA cluster. In Nocardia uniformis, NocE is a protein of unknown function encoded by a gene (nocE) located in the nocardicin cluster (Gunsinor et al. 2004). The second gene, Sfla_0551, encodes a small protein (93 amino acids) annotated as a transportrelated protein but with no correspondence to any protein required for clavulanic acid biosynthesis. The third gene inserted in the cluster, Sfla 0553, encodes a 335 amino acid protein $51 \%$ identical to SCLAV 4203, a protein of unknown function encoded by orf11 which in S. clavuligerus is located in the cephamycin $\mathrm{C}$ gene cluster. It is unclear if the presence of these genes affects CA production in S. flavogriseus and the elucidation will require studies using deleted mutants.
Three CA biosynthesis-unrelated genes, Svir_ 33330, Svir_33340, and Svir_33350, are inserted in the CA cluster of Sac. viridis (Fig. 1b); they encode, respectively, a 625-amino acid penicillin-binding protein with putative transpeptidase activity, a 1,414-amino acid hypothetical protein with some similarity to NocE, and an 88-amino acid hypothetical protein.

Expression of the CA gene cluster and clavulanic acid production using cultures of $S$. flavogriseus

Due to the lack of a regulatory ccaR gene in Sac. viridis genome and since initial tests showed that Sac. viridis grows poorly in our standard media and growth conditions, we decided to focus in S. flavogriseus to study clavulanic gene expression.

Both S. clavuligerus and S. flavogriseus were grown in parallel cultures in nine different media, the complex YEME, MS, R5, MEY, TBO, ISP4, and TSB media and the defined MG and SA media under the normal growth conditions used for S. clavuligerus. While $S$. clavuligerus produced CA with different yields in all the media, no detectable production of CA by S. flavogriseus was observed at any time using bioassays or by HPLC analysis of broth of the liquid cultures. Supplementation of the $S$. flavogriseus cultures with the CA precursors glycerol $0(2 \% v / v)$, arginine $(5$ or $10 \mathrm{mM})$, or both did not result in CA production (data not shown). 
To test whether the lack of CA production was due to the absence of transcription, RNA was purified from $S$. flavogriseus cultures after incubation in YEME, R5, TSB, and SA media for 24 and $48 \mathrm{~h}$, and RT-PCR analyses were performed for the biosynthetic genes and some other genes essential for CA production using oligonucleotides specific for $S$. flavogriseus genes (see Table S1). Positive and negative PCR controls (not shown) confirmed that the sets of oligonucleotides used were effective in amplification and discriminate between $S$. clavuligerus and $S$. flavogriseus CA orthologous genes.

The results of the $S$. flavogriseus RT-PCR reactions are shown in Fig. 2. A clear expression of the ccaR and claR regulatory genes was observed, especially in YEME, R5, and SA media that suggested that the genes for the early and late steps of the pathway activated using these regulators might be also expressed. However, the expression of $\operatorname{orf12}$, orf14, and orf16 in S.flavogriseus was low or undetectable in all the tested media, and the expression of cyp, car, and cas 2 was low (data not shown), even after 35 amplification cycles. The low expression of some biosynthesis genes and the undetectable levels of others could explain the lack of CA production using $S$. flavogriseus in the culture conditions tested.

The $c c a R_{\mathrm{F}}$ gene of $S$. flavogriseus and the $c c a R_{\mathrm{C}}$ of $S$. clavuligerus are not functional in the heterologous strains

Although the endogenous $c c a R$ gene (named $c c a R_{\mathrm{F}}$ thereafter) was expressed in S. flavogriseus ATCC 33331, as shown by RT-PCR (see Fig. 2), the protein might be present at low levels or might not be functional what could explain the silent nature of $S$. flavogriseus CA cluster. To eliminate these hypotheses,

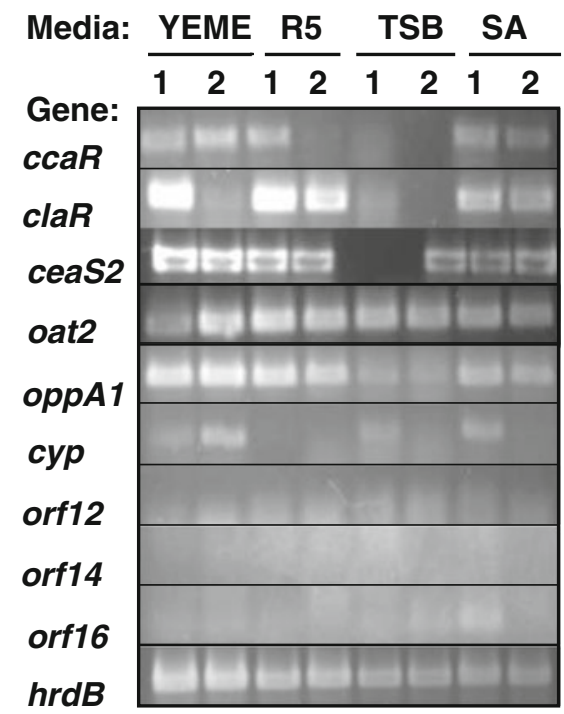

Fig. 2 Expression of the CA biosynthesis genes as detected by RT-PCR. Isolated RNA from $S$. flavogriseus cultures grown in YEME, R5, TSB, and SA media for $24 \mathrm{~h}$ (1) and $48 \mathrm{~h}$ (2) was submitted to 35 cycles of amplification for the genes indicated at the left side of the figure. The $h r d B$ gene is used as external control we introduced the $S$. clavuligerus ccaR gene (that will be named $c c a R_{\mathrm{C}}$ ) expressed from the $\mathrm{P}_{\text {fur }}$ promoter in $S$. flavogriseus ATCC 33331 by conjugation. The strain obtained, S. flavogriseus:: $\left[\mathrm{P}_{\text {fur }}-c c a R_{\mathrm{C}}\right]$, expressed $c c a R_{\mathrm{C}}$ as detected by RT-PCR but did not produce clavulanic acid in any media (data not shown), suggesting that $c c a R_{\mathrm{C}}$ is not functional in $S$. flavogriseus, perhaps due to the lack of conserved heptameric CcaR-binding sequences in the putative target promoters.

To determine whether the $S$. flavogriseus $c c a R_{\mathrm{F}}$ was able to complement $c c a R_{\mathrm{C}}$ in the disrupted mutant $S$. clavuligerus $\Delta c c a R:$ :tsr, unable to produce clavulanic acid or cephamycin C, $c c a R_{\mathrm{F}}$ (under the $\mathrm{P}_{\text {fur }}$ promoter) was introduced in $S$. clavuligerus $\Delta$ ccaR::tsr. Seven exconjugants of $S$. clavuligerus $(\Delta c c a R:: t s r)::\left[\mathrm{P}_{\text {fur }}-c c a R_{\mathrm{F}}\right]$ were grown in solid and liquid TSB, MEY, and TBO media for up to $60 \mathrm{~h}$. However, neither cephamycin $\mathrm{C}$ nor clavulanic acid was detected in any conditions in spite of the positive expression of $c c a R_{\mathrm{F}}$, as detected by RT-PCR. This result suggests that the $\mathrm{CcaR}_{\mathrm{F}}$ protein is unable to complement the lack of $\mathrm{CcaR}_{\mathrm{C}}$ in the $S$. clavuligerus disrupted mutant, perhaps because $\mathrm{CcaR}_{\mathrm{F}}$ does not recognize promoters essential for clavulanic acid biosynthesis located in the CA gene cluster of $S$. clavuligerus. Positive controls complementing the $\Delta c c a R$ S. clavuligerus strain with $c c a R_{\mathrm{C}}$ proved that the homologous regulatory protein complements the CA production as reported previously (Pérez-Llarena et al. 1997a, 1997b)

Heterologous expression of $S$. clavuligerus clavulanic acid cluster in S. flavogriseus ATCC 33331 and S. coelicolor M1146

Since the endogenous CA cluster of $S$. flavogriseus was not functional and $c c a R_{\mathrm{C}}$ did not improve CA production, we decided to study the heterologous expression of the complete $S$. clavuligerus CA cluster (except the $c c a R$ gene that is in the cephamycin C cluster) in S. flavogriseus ATCC 33331 and in the model organism $S$. coelicolor M1146 to analyze the influence of the genetic background of these strains on the CA cluster expression. The whole cluster for clavulanic acid, present in cosmid [SCos-CA], was introduced by conjugation in these strains and in derived strains carrying the $c c a R_{\mathrm{C}}$ gene expressed from the $\mathrm{P}_{\text {fur }}$ promoter; the strains obtained were named $S$. flavogriseus::[SCos-CA], S. flavogriseus::[ $\mathrm{P}_{\text {fur }}-$ ccaR $\left.\mathrm{C}\right]::[\mathrm{SCos}-$

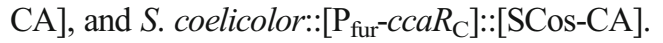

These strains and their controls lacking [SCos-CA], were tested in the nine solid media previously described. No production of CA was ever detected in the $S$. coelicolor exconjugants (data not shown). However, a clear inhibition zone produced by CA was observed in plugs of $S$. flavogriseus::[SCos-CA] and $S$. flavogriseus::[ $\left.\mathrm{P}_{\text {fur }}-c c a R_{\mathrm{C}}\right]::[\mathrm{SCOS}-\mathrm{CA}]$ (Fig. 3 inset) but not in the control $S$. flavogriseus grown in MS, ISP4, and particularly in MEY and TBO media.

To validate these results, two exconjugants of $S$. flavogriseus::[SCos-CA] and S. flavogriseus::[ $\left.\mathrm{P}_{\text {fur }}-c c a R_{\mathrm{C}}\right]::[\mathrm{SCos}-$ 
Fig. 3 Heterologous clavulanic acid production using exconjugants of S. flavogriseus. Growth (left panels) and clavulanic acid production (right panels) in MEY (a) and TBO medium (b) of $S$. flavogriseus ATCC 33331 (open triangles), S. flavogriseus:: $\left[\mathrm{P}_{\text {fur }}-c c a R_{\mathrm{C}}\right]$ (open squares), S. flavogriseus::[SCos$\mathrm{CA}]$ (black triangles), and $S$.

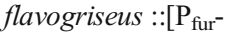
cca $\left._{\mathrm{C}}\right]::[\mathrm{SCos}-\mathrm{CA}]$ (black squares). Inset in the lower-right panel: bioassays of broth samples from S. flavogriseus ATCC 33331 (1) and S. flavogriseus::[SCos$\mathrm{CA}]$ (2) cultures grown in TBO medium for $48 \mathrm{~h}$

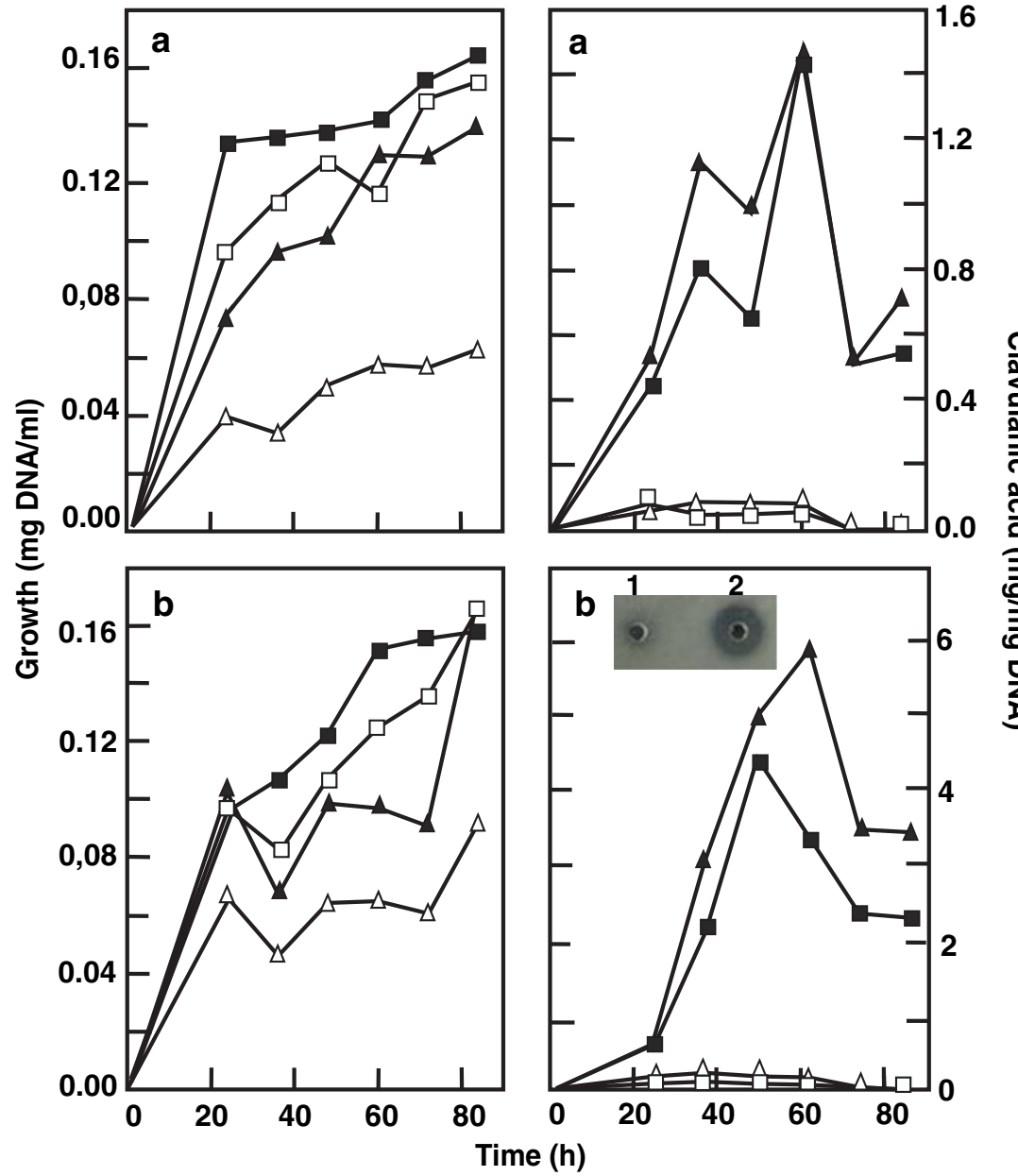

CA] were separately grown in MEY and TBO liquid cultures using cultures of $S$. clavuligerus ATCC 27064 and S. flavogriseus ATCC 33331 as controls (Fig. 3). The growth of the strains was relatively similar with the exception of S. flavogriseus ATCC 33331 that grew more slowly. The MEY medium supported a faster growth with top growth values at $45 \mathrm{~h}$ of cultivation, while in TBO, the maximum growth value was reached at $70 \mathrm{~h}$. Production of clavulanic acid was particularly high in TBO medium with values of 4.5 to $6 \mu \mathrm{g} \mathrm{CA} /$ mg DNA $(0.6 \mu \mathrm{g} / \mathrm{ml})$ at $45 \mathrm{~h}$, about four- to fivefold higher than in MEY medium. These values were, however, lower than those of $S$. clavuligerus ATCC 27064 grown in the same medium and conditions (not shown) which reached maximal values of 87 and $750 \mu \mathrm{g} / \mathrm{mg}$ DNA (28.5 and $164.5 \mu \mathrm{g} / \mathrm{ml}$ ) in MEY and TBO media, respectively. The inhibition zone on penicillin-resistant Klebisella bioassays produced using broths of the cultures is shown in the inset of Fig. 3.

Taken together, these results indicate that the $S$. clavuligerus CA genes (except $c c a R$ ) are functional in $S$. flavogriseus. Therefore, the lack of CA production in S. flavogriseus is due to lack of CA biosynthesis enzymes.
Confirmation by HPLC-MS that the product is clavulanic acid

To further confirm that the product responsible for the inhibition zones was clavulanic acid, the broth of cultures of two $S$. flavogriseus::[SCos-CA] exconjugants was analyzed by HPLC. A small peak was detected with the same retention time as authentic clavulanic acid (not shown). These broths were also analyzed by HPLC-MS. By electrospray ionization in the negative mode and selected ion recording at $\mathrm{m} / \mathrm{z} 198$, a peak eluting at 14 min was detected in both the standard and the cultures of two exconjugants (Fig. 4A,B). In both cases, mass spectra of that peak showed a negative ion, $[\mathrm{M}-\mathrm{H}]^{-}$, with an $m / z$ value of 198, as expected for clavulanic acid (Fig. 4C).

Expression of the endogenous and heterologous clavulanic acid gene clusters in S. flavogriseus as determined by RT-qPCR

To further investigate the lack of clavulanic acid formation in S. flavogriseus ATCC 33331, we compared by RT-qPCR the expression of the clavulanic acid genes of S. flavogriseus ATCC 33331 and S. clavuligerus ATCC 27064. In addition, 
Fig. 4 Mass chromatography obtained by selected ion recording at $m / z$ 198. a Clavulanic acid standards $(0.1 \mu \mathrm{g})$; b broth $(10 \mu \mathrm{l})$ from a 60 -h culture of $S$. flavogriseus::[SCos-CA] grown in TBO medium. c Mass spectra of the clavulanic acid peak at chromatogram $\mathrm{B}$ detected in negative mode $(\mathrm{M}-\mathrm{H})$
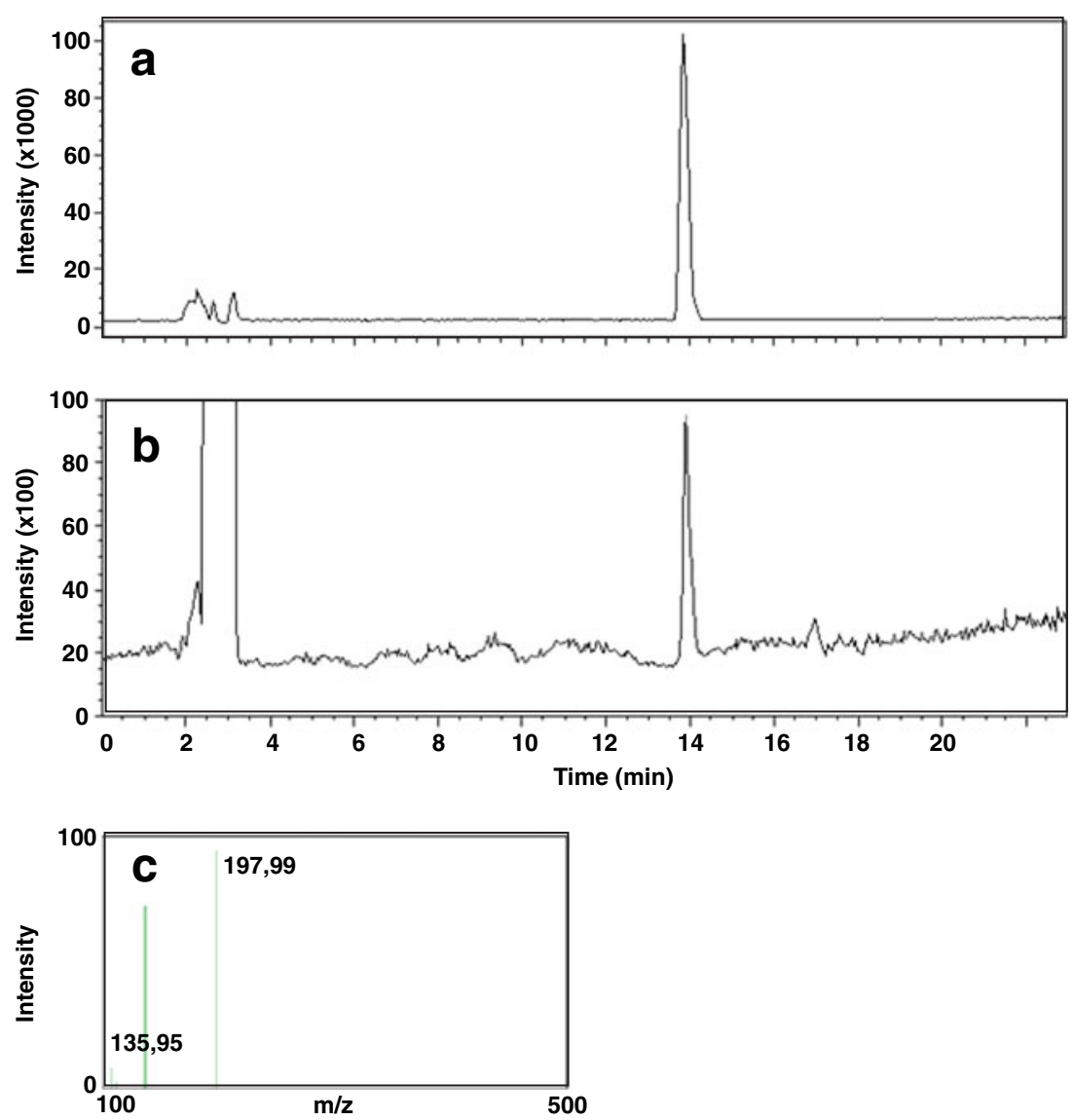

expression of both the endogenous and the heterologous CA gene clusters were studied (using specific sets of oligonucleotides) in S. flavogriseus::[SCos-CA] which carries the CA cluster of $S$. clavuligerus. The studies were done at $48 \mathrm{~h}$ of growth in liquid TBO medium which support high CA formation using all the strains.

All the CA genes were well expressed in control $S$. clavuligerus ATCC 27064 (Fig. 5A) with expression values between 4 (ceaS, car) and 6 (oppA1). Interestingly, several genes of the endogenous CA cluster of $S$. flavogriseus ATCC 33331 (not shown) and S. flavogriseus::[SCos-CA] (Fig. 5B, black bars) were expressed with a similar pattern but at lower intensities in both $S$. flavogriseus strains. All the endogenous genes for the early steps of the pathway (ceaS2 to cas2) were expressed in S. flavogriseus::[SCos-CA] with expression values of about 2 to 2.3, while oat2, oppA1, and gcaS reached values of 4 to 4.6. These expression values, even though lower than those of $S$. clavuligerus (Fig.5A), should be high enough to support CA formation in S. flavogriseus. However, endogenous genes essential for CA formation as cyp, orf12, orf13, orf14, or oppA2 were barely or not expressed (expression values around 0.01 ) in S. flavogriseus or S. flavogriseus::[SCos-CA]. The low expression of these genes, which might have an accumulative negative effect, is the most plausible explanation of the lack of clavulanic acid production in this strain. All S. clavuligerus original genes are poorly expressed in the recombinant $S$. flavogriseus::[SCos-CA] (without the S. clavuligerus ccaR gene) (Fig. 5B, white bars) with average values of expression of 0.3 to 0.4 , which was about 11- to 14-fold lower than in their $S$. clavuligerus natural host; however, there was an expression of the exogenous cyp, orf12, orf13, or oppA2 genes that although small, still was three- to fourfold higher than in $S$. flavogriseus ATCC 33331. This expression of the heterologous genes explains the formation of CA by the recombinant $S$. flavogriseus::[SCos-CA] which in $\mathrm{TBO}$ reaches $0.6 \mu \mathrm{g} / \mathrm{ml}$ compared to the $164.5 \mu \mathrm{g} / \mathrm{ml}$ produced by $S$. clavuligerus in the same medium. The absence of heterologous expression in $S$. coelicolor and the low expression in S. flavogriseus illustrate the important role of the rest of the genes (i.e., the genetic background) on the expression of the CA cluster.

\section{Discussion}

Streptomyces species are prolific producers of antibiotics and other related secondary metabolites (Martín et al. 2000; Bérdy 2012). The availability of an increasing number of genome sequences of species of Streptomyces (Pati et al. 2009; Ikeda 
Fig. 5 Quantitative expression by RT-qPCR of the clavulanic acid biosynthesis genes. A White bars show the expression of the genes (indicated at the bottom of the figure) in S. clavuligerus grown in TBO medium after $48 \mathrm{~h}$ of culture. B Expression of the clavulanic acid biosynthesis genes in S. flavogriseus::[SCosCA]. Expression of genes of the endogenous $S$. flavogriseus cluster is shown in black bars. Heterologous expression of genes of $S$. clavuligerus CA cluster in $S$. flavogriseus::[SCos-CA] is shown in white bars

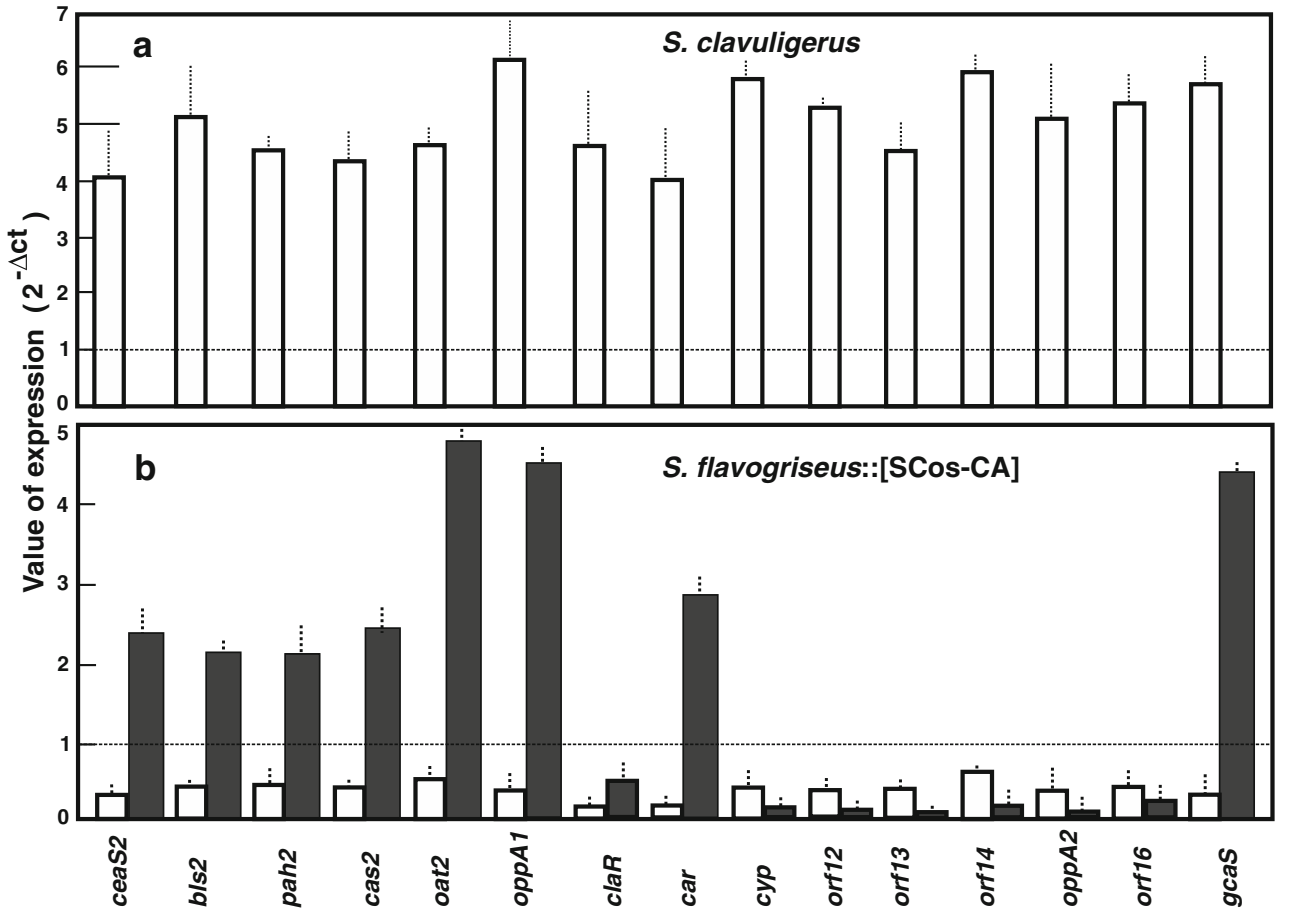

et al. 2003; Bentley et al. 2002; Barreiro et al. 2012, among others) has provided evidence for the presence of about 20 to 30 gene clusters for secondary metabolites in each of the genomes (Bentley et al. 2002; Medema et al. 2010). However, the presence of these gene clusters does not mean that all of them are expressed (Laureti et al. 2011) Many secondary metabolites gene clusters may be silent or expressed only at very low levels under the culture conditions used in the laboratory (Brakhage and Schroeckh 2011), but they might be expressed in natural environment resulting in an ecological advantage for the strain. An important challenge is to understand why silent clusters are silent and to modify the genes to achieve their expression.

In this work, we report the presence of a complete clavulanic acid gene cluster in two actinomycetes, S. flavogriseus and Sac. viridis. The organization of CA genes in these genomes shows gene conservation in specific subclusters similar to those of $S$. clavuligerus; however, these subclusters are rearranged in a patchwork-like organization, indicating that reorganization of these blocks has occurred during evolution of the clusters probably associated with horizontal transfer phenomena. We found previously a similar patchwork-like arrangement of the cephamycin C gene clusters in Amycolatopsis (formerly Nocardia) lactamdurans and S. clavuligerus (Liras et al. 1998; Enguita et al. 1998). It is very interesting that the genes of block A for the early steps of CA biosynthesis are also required for clavam biosynthesis in $S$. antibioticus, a strain that does not produce clavulanic acid and lacks blocks B and C (Nobary and Jensen 2012). These observations suggest that the full CA pathway has been evolutively assembled by combining an early pathway (up to clavaminic acid formation, block A) common to the biosynthesis of antifungal clavams and the present "late" biosynthesis pathway of CA (block B).

An important difference between the CA cluster in $S$. clavuligerus and $S$. flavogriseus is the presence in the later of the $c c a R$ gene, encoding a SARP-type regulator (block $\mathrm{C}$ in Fig. 1b) (Pérez-Llarena et al. 1997a). These differences suggest that intense reorganization processes have occurred within the genus Streptomyces that cannot be explained by simple rearrangements during vertical inheritance of the CA genes from a common Streptomyces ancestor.

S. flavogriseus did not produce CA in eight different culture media, in which S. clavuligerus produces high levels, and therefore, the $S$. flavogriseus CA cluster might be considered as silent. Several of the S. flavogriseus CA biosynthesis genes analyzed by RT-PCR were found to be expressed at different degrees depending on the media but always at low levels as reflected by the requirement of a high number of PCR cycles needed to detect their expression. RT-qPCR studies allowed to identify which genes of the CA cluster are really silent. They are cyp, orf12, orf13, orf14, and oppA2, all known to be essential for CA formation in S. clavuligerus (Mellado et al. 2002; Li et al. 2000; Lorenzana et al. 2004). Some of these genes are underexpressed in the absence of CcaR (Santamarta et al. 2011). The high expression of the genes for the early steps of the pathway and the poor expression of other genes suggest that $S$. flavogriseus is in an intermediate evolutive stage and might lose eventually the functionality of the genes for the late steps, leading to a strain similar to $S$. antibioticus (Nobary and Jensen 2012). 
Heterologous expression of particular genes to increase antibiotic production is a common strategy. Moreover, the heterologous expression of complete gene clusters is a new approach for novel antibiotic production based on the analysis of gene clusters located in rare actinomycetes (Tong et al. 2013) or in Streptomyces with unstable antibiotic gene expression levels. Specific $S$. coelicolor host strains have been constructed with this purpose (Gomez-Escribano and Bibb 2011). In some cases, as that of aminocoumarins or trithiazolylpyridine-containing derived compounds, the heterologous expression in genetically modified strains of $S$. coelicolor and S. lividans is satisfactory (Eustáquio et al. 2005; Flinspach et al. 2010; Young and Walsh 2011) but other gene clusters, as that of holomycin, are poorly expressed (Huang et al. 2011; Robles-Reglero et al. 2013). When the $S$. clavuligerus CA cluster was introduced in $S$. flavogriseus, the genes were expressed although at very low levels, as detected by RT-qPCR, independently of the presence of the $S$. clavuligerus regulatory activator $\mathrm{CcaR}_{\mathrm{C}}$. Indeed, the introduction of the $S$. clavuligerus regulatory ccaRC gene, expressed from the $\mathrm{P}_{\text {fur }}$ promoter, does not improve significantly the low production of CA in S. flavogriseus.

An interesting question is why some genes of the $S$. flavogriseus CA gene cluster are not expressed since both the $c c a R$ and claR regulatory activators are well expressed in this species (Fig. 2). The simplest explanation is that the promoter regions of those genes have evolved in S. flavogriseus being unable to make stable interactions with the RNA polymerase and the $\mathrm{CcaR}$ or ClaR positive regulators. A similar situation has been found with a 19-kb thienamycin-like cluster located $41 \mathrm{~kb}$ away from the CA cluster in S. flavogriseus. The thienamycin cluster, although apparently complete, is silent in several media (Blanco 2012) although there are no expression studies available.

S. flavogriseus is unable to use the heterologous $\mathrm{CcaR}_{\mathrm{C}}$ regulatory protein of $S$. clavuligerus for the expression of its own CA genes when introduced in plasmid pMS82. Moreover, the $S$. flavogriseus $\mathrm{CcaR}_{\mathrm{F}}$ protein activates expression of its own (homologous) CA genes in the wild-type $S$. flavogriseus to a lower degree than in S. clavuligerus. The lack of effect of $\mathrm{CcaR}_{\mathrm{C}}$ on the $S$. flavogriseus $\mathrm{CA}$ genes might be explained by the lack of detectable heptameric sequences for $\mathrm{CcaR}_{\mathrm{C}}$ binding (Santamarta et al. 2011) in the promoter regions of the $S$. flavogriseus CA biosynthesis genes.

The low degree of activation of the $S$. clavuligerus CA genes by $\mathrm{CcaR}_{\mathrm{C}}$, when the structural genes and the $\mathrm{CcaR}_{\mathrm{C}}$ regulator (both from S. clavuligerus) are introduced in S. flavogriseus, suggests that a molecule required for full CcaR-mediated induction of homologous CA genes is deficient (at least partially) in $S$. flavogriseus. This might be a still-unknown ligand required for full CcaR activity as occur in other Streptomyces species (Wang et al. 2009; Xu et al. 2010). Interestingly, the constructions carrying $\left[\mathrm{P}_{\mathrm{fur}}-c c a R_{\mathrm{C}}\right]$ and $[\mathrm{SCos}-\mathrm{CA}]$ result in heterologous production of clavulanic acid in S. flavogriseus but not in $S$. coelicolor. This might reflect differences in the genetic background in both host microorganisms, with $S$. coelicolor phylogenetically much more distant in relation to $S$. clavuligerus than S. flavogriseus (Zhou et al. 2011). We propose that in many cases, heterologous expression may be limited by the lack of appropriate pools of precursors (as a result of different biosynthetic steps) or of unknown ligands or protein modifying systems that may decrease or even prevent the expression of an apparently complete gene cluster. Indeed, the availability of arginine, a well-known precursor of CA (Valentine et al. 1993; Romero et al. 1986), is probably affected by the very different organization of the arg gene clusters in $S$. clavuligerus and $S$. coelicolor (Rodríguez-García et al. 1997). Heterologous expression is easier if the host used has a very similar or at least a related pathway providing adequate precursors (e.g., rare amino acid biosynthetic pathways for heterologous expression of a non-ribosomal peptide compounds). This is the case of $S$. flavogriseus as host for the heterologous expression of CA genes when compared with S. coelicolor.

Acknowledgments This work was supported by grants BIO2009-09820 and LE046A11-2 from the Spanish Ministry of Economy and Competitivity and the Junta de Castilla y León, respectively. R. Álvarez-Álvarez and Y. Martínez-Burgo received PFU fellowships from the Spanish Ministry of Education, Culture and Sports. We appreciate the collaboration of Dr. T. López-García in the design of oligonucleotides and RT-qPCR experiments and the reception of plasmid pFL1272 from Dr. F. Lombo.

\section{References}

Aidoo KA, Wong A, Alexander DC, Rittammer RA, Jensen SE (1994) Cloning, sequencing and disruption of a gene from Streptomyces clavuligerus involved in clavulanic acid biosynthesis. Gene 147:4146

Aigle B, Wietzorrek A, Takano E, Bibb MJ (2000) A single amino acid substitution in region 1.2 of the principal sigma factor of Streptomyces coelicolor A3(2) results in pleiotropic loss of antibiotic production. Mol Microbiol 37:995-1004

Alexander DC, Jensen SE (1998) Investigation of the Streptomyces clavuligerus cephamycin $\mathrm{C}$ gene cluster and its regulation by the CcaR protein. J Bacteriol 180:4068-4079

Arulanantham H, Kershaw NJ, Hewitson KS, Hughes CE, Thirkettle JE, Schofield CJ (2006) ORF17 from the clavulanic acid biosynthesis gene cluster catalyzes the ATP-dependent formation of $N$-glycylclavaminic acid. J Biol Chem 6:279-287

Bachmann BO, Li R, Townsend CA (1998) Beta-Lactam synthetase: a new biosynthetic enzyme. Proc Natl Acad Sci USA 95:9082-9086

Barreiro C, Prieto C, Sola-Landa A, Solera E, Martínez-Castro M, PérezRedondo R, García-Estrada C, Aparicio JF, Fernández-Martínez LT, Santos-Aberturas J, Salehi-Najafabadi Z, Rodríguez-García A, Tauch A, Martín JF (2012) Draft genome of Streptomyces tsukubaensis NRRL 18488, the producer of the clinically important immunosuppressant Tacrolimus (FK506). J Bacteriol 174:3756-3757

Bentley SD, Chater KF, Cerdeño-Tárraga AM, Challis GL, Thomson NR, James KD, Harris DE, Quail MA, Kieser H, Harper D, Bateman A, Brown S, Chandra G, Chen CW, Collins M, Cronin A, Fraser A, Goble A, Hidalgo J, Hornsby T, Howarth S, Huang CH, Kieser T, Larke L, Murphy L, Oliver K, O'Neil S, Rabbinowitsch E, Rajandream MA, 
Rutherford K, Rutter S, Seeger K, Saunders D, Sharp S, Squares R, Squares S, Taylor K, Warren T, Wietzorrek A, Woodward J, Barrell BG, Parkhill J, Hopwood DA (2002) Complete genome sequence of the model actinomycete Streptomyces coelicolor A3(2). Nature 417:141-147

Bérdy J (2012) Thoughts and facts about antibiotics: where we are now and where we are. J Antibiot 65:385-395

Bignell DR, Tahlan K, Colvin KR, Jensen SE, Leskiw BK (2005) Expression of $c c a R$, encoding the positive activator of cephamycin $\mathrm{C}$ and clavulanic acid production in Streptomyces clavuligerus, is dependent on bldG. Antimicrob Agents Chemother 49:1529-1541

Blanco G (2012) Comparative analysis of a cryptic thienamycin-like gene cluster identified in Streptomyces flavogriseus by genome mining. Arch Microbiol 194:549-555

Brakhage AA, Schroeckh V (2011) Fungal secondary metabolites - strategies to activate silent gene clusters. Fungal Genet Biol 48:15-22

Doull JL, Vining LC (1989) Culture conditions promoting dispersed growth and biphasic production of actinorhodin in shaken cultures of Streptomyces coelicolor A3(2). FEMS Microbiol Lett 3:265-268

Enguita FJ, Coque JJ, Liras P, Martín JF (1998) The nine genes of the Nocardia lactamdurans cephamycin cluster are transcribed into large mRNAs from three promoters, two of them located in a bidirectional promoter region. J Bacteriol 180:5489-5494

Eustáquio AS, Gust B, Galm U, Li SM, Chater KF, Heide L (2005) Heterologous expression of novobiocin and clorobiocin biosynthetic gene clusters. Appl Environ Microbiol 71:2452-2459

Flinspach K, Westrich L, Kaysser L, Siebenberg S, Gomez-Escribano JP, Bibb M, Gust B, Heide L (2010) Heterologous expression of the biosynthetic gene clusters of coumermycin $\mathrm{A}(1)$, clorobiocin and caprazamycins in genetically modified Streptomyces coelicolor strains. Biopolymers 93:823-832

Foulstone M, Reading C (1982) Assay of amoxicillin and clavulanic acid, the components of Augmentin, in biological fluids with high-performance liquid chromatography. Antimicrob Agents Chemoter 22:753-762

Gomez-Escribano JP, Bibb MJ (2011) Engineering Streptomyces coelicolor for heterologous expression of secondary metabolite gene clusters. Microb Biotechnol 4:207-215

Gunsinor M, Breazeale SD, Lind AJ, Ravel J, Janc JW, Townsend CA (2004) The biosynthetic gene cluster for a monocyclic beta-lactam antibiotic, nocardicin A. Chem Biol 11:927-938

Higgens CE, Hamill RL, Sands TH, Hoehn MM, Davis NE, Najarahan R, Boeck LD (1974) The occurrence of desacetoxycephalosporin C in fungi and Streptomyces. J Antibiot 27:298-300

Huang S, Yudong Z, Zhiwei Q, Xiaoling W, Mayca O, Chen L, He J, Yu Y, Deng H (2011) Identification and heterologous expression of the biosynthetic gene cluster for holomycin produced by Streptomyces clavuligerus. Process Biochem 46:811-816

Ikeda H, Ishikawa J, Hanamoto A, Shinose M, Kikuchi H, Shiba T, Sakaki Y, Hattori M, Omura S (2003) Complete genome sequence and comparative analysis of the industrial microorganism Streptomyces avermitilis. Nat Biotechnol 21:526-531

Ishida K, Hung TV, Liou K, Lee HC, Shin CH, Sohng JK (2006) Characterization of $p b p A$ and $p b p 2$ encoding penicillin-binding proteins located on the downstream of clavulanic acid gene cluster in Streptomyces clavuligerus. Biotechnol Lett 28:409-417

Janc JW, Egan LA, Townsend CA (1995) Purification and characterization of clavaminate synthase from Streptomyces antibioticus. A multifunctional enzyme of clavam biosynthesis. J Biol Chem 270:5399-5404

Khaleeli N, Li R, Townsend CA (1999) Origin of the $\beta$-lactam carbons in clavulanic acid from an usual thiamine pyrophosphate-mediated reaction. J Am Chem Soc 121:9223-9224

Kieser T, Bibb MJ, Buttner MJ, Chater KF, Hopwood DA (2000) Practical Streptomyces Genetics. John Innes Foundation, Norwich

Kurt A, Álvarez-Alvarez R, Liras P, Özcengiz G (2013) Role of the $\mathrm{cmcH}$ $c c a R$ intergenic region and ccaR overexpression in cephamycin $\mathrm{C}$ biosynthesis in Streptomyces clavuligerus. Applied Microbiol Biotechnol. doi: 10.1007/s00253-013-4721-4724

Laureti L, Song L, Huang S, Corre C, Leblond P, Challis GL, Aigle B (2011) Identification of a bioactive 51-membered macrolide complex by activation of a silent polyketide synthase in Streptomyces ambofaciens. Proc Natl Acad Sci USA 108:6258-6263

Li R, Khaleeli N, Townsend CA (2000) Expansion of the clavulanic acid gene cluster: identification and in vivo functional analysis of three new genes required for biosynthesis of clavulanic acid by Streptomyces clavuligerus. J Bacteriol 182:4087-4095

Liras P, Rodríguez-García A, Martín JF (1998) Evolution of the clusters of genes for $\beta$-lactam antibiotics: a model for evolutive combinatorial assembly of new $\beta$-lactams. Internat Microbiol $1: 271-278$

Liras P, Santamarta I, Pérez-Redondo R (2011) Clavulanic acid and clavams biosynthesis and regulation. In: Dyson P (ed) Streptomyces Molecular Biology and Biotechnology. Caister Academic Press, Norfolk, UK, pp $167-178$

Livak KJ, Schmittgen TD (2001) Analysis of relative gene expression data using real time quantitative PCR and the 22DDCt Method. Methods 25:402-408

López-García MT, Santamarta I, Liras P (2010) Morphological differentiation and clavulanic acid formation are affected in a Streptomyces clavuligerus adpA-deleted mutant. Microbiology 156:2354-2365

Lorenzana LM, Pérez-Redondo R, Santamarta I, Martín JF, Liras P (2004) Two oligopeptide-permease-encoding genes in the clavulanic acid cluster of Streptomyces clavuligerus are essential for production of the beta-lactamase inhibitor. J Bacteriol 186:3431-3438

Marsh EN, Chang MD, Townsend CA (1992) Two isozymes of clavaminate synthase central to clavulanic acid formation: cloning and sequencing of both genes from Streptomyces clavuligerus. Biochemistry $31: 12648-12657$

Martín JF, Liras P (2010) Engineering of Regulatory Cascades and Networks Controlling Antibiotic Biosynthesis in Streptomyces. Current Op in Microbiol 13:263-273

Martín JF, Gutiérrez S, Aparicio JF (2000) Secondary metabolites. In: Lederberg J (ed) Encyclopedia of Microbiology, vol 4, 2nd edn. Academic Press, San Diego, pp 213-236

Matthew AG, Till R, Smith MCM (2003) Integration site for Streptomyces phage $\Phi \mathrm{BT} 1$ and development of site-specific integrating vectors. J Bacteriol 185:5320-5323

Medema MH, Trefzer A, Kovalchuk A, van den Berg M, Müller U, Heijne W, Wu L, Alam MT, Ronning CM, Nierman WC, Bovenberg RA, Breitling R, Takano E (2010) The sequence of a $1.8-\mathrm{mb}$ bacterial linear plasmid reveals a rich evolutionary reservoir of secondary metabolic pathways. Genome Biol Evol 2:212-224

Mellado E, Lorenzana LM, Rodríguez-Sáiz M, Díez B, Liras P, Barredo JL (2002) The clavulanic acid biosynthetic cluster of Streptomyces clavuligerus: genetic organization of the region upstream of the car gene. Microbiology 148:1427-1438

Nicholson NH, Baggaley KH, Cassels R, Davison M, Elson SW, Fulston M, Tyler JW, Woroniecki ST (1994) Evidence that the intermediate biosynthetic precursor of clavulanic acid is its $\mathrm{N}$-aldehyde analogue. J Chem Soc Chem Commun 1994:1281-1282

Nobary GS, Jensen SE (2012) A comparison of the clavam biosynthetic gene clusters in Streptomyces antibioticus Tü1718 and Streptomyces clavuligerus. Can J Microbiol 58:413-425

Ortiz de Orue Lucana D, Tröller M, Schrempf H (2003) Amino acid residues involved in reversible thiol formation and zinc ion binding in the Streptomyces reticuli redox regulator FurS. Mol Genet Genomics 268:618-627

Paradkar AS, Aidoo KA, Jensen SE (1998) A pathway-specific transcriptional activator regulates late steps of clavulanic acid biosynthesis in Streptomyces clavuligerus. Mol Microbiol 27:831-843 
Pati A, Sikorski J, Nolan M, Lapidus A, Copeland A, Glavina del Rio T, Lucas S, Chen F, Tice H, Pitluck S, Cheng JF, Chertkov O, Brettin T, Han C, Detter JC, Kuske C, Bruce D, Goodwin L, Chain P, D'haeseleer P, Chen A, Palaniappan K, Ivanova N, Mavromatis K, Mikhailova N, Rohde M, Tindall BJ, Göker M, Bristow J, Eisen JA, Markowitz V, Hugenholtz P, Kyrpides NC, Klenk HP (2009) Complete genome sequence of Saccharomonospora viridis type strain (P101). Stand Genomic Sci 1:141-149

Pérez-Llarena F, Martín JF, Galleni M, Coque JJ, Fuente JL, Frère JM, Liras P (1997a) The bla gene of the cephamycin cluster of Streptomyces clavuligerus encodes a class A beta-lactamase of low enzymatic activity. J Bacteriol 179:6035-6040

Pérez-Llarena FJ, Liras P, Rodríguez-García A, Martín JF (1997b) A regulatory gene $(c c a \mathrm{R})$ required for cephamycin and clavulanic acid production in Streptomyces clavuligerus: amplification results in overproduction of both B-lactam compounds. J Bacteriol 179:20532059

Pérez-Redondo R, Rodríguez-García A, Martín JF, Liras P (1998) The cla $\mathrm{R}$ gene of Streptomyces clavuligerus, encoding a LysR-type regulatory protein controlling clavulanic acid biosynthesis, is linked to the clavulanate-9-aldehyde reductase ( $c a r$ gene. Gene 211:311321

Pérez-Redondo R, Rodríguez-García A, Martín JF, Liras P (1999) Deletion of the $p y c$ gene blocks clavulanic acid biosynthesis except in glycerol-containing medium: evidence for two different genes in formation of the C3 unit. J Bacteriol 181:6922-6928

Robles-Reglero V, Santamarta I, Álvarez-Álvarez R, Martín JF, Liras P (2013) Transcriptional analysis and proteomics of the holomycin gene cluster in overproducer mutants of Streptomyces clavuligerus. J Biotechnol 163(1):69-76

Rodríguez-García A, Ludovice M, Martín JF, Liras P (1997) Arginine boxes and the $\arg R$ gene in Streptomyces clavuligerus: evidence for a clear regulation of the arginine pathway. Mol Microbiol 25:219-28

Romero J, Liras P, Martín JF (1984) Dissociation of cephamycin and clavulanic acid biosynthesis in Streptomyces clavuligerus. Appl Microbiol Biotechnol 20:318-325
Romero J, Liras P, Martín JF (1986) Utilization of ornithine and arginine as specific precursors of clavulanic acid. Appl Environ Microbiol 52:892 897

Santamarta I, López-García MT, Kurt A, Nárdiz N, Pérez-Redondo R, Álvarez-Álvarez R, Martín JF, Liras P (2011) Characterization of DNA-binding sequences for $\mathrm{CcaR}$ in the cephamycin-clavulanic acid supercluster of Streptomyces clavuligerus. Mol Microbiol 81:968-981

Schmittgen TD, Zakrajsek BA (2000) Effect of experimental treatment on housekeeping gene expression: validation by real-time, quantitative RT-PCR. J Biochem Biophys Methods 46:69-81

Tong L, Yuanyuan D, Qiu C, Jingtao Z, Weiming Z, Kui H, Wenli L (2013) Cloning, characterization and heterologous expression of the indolocarbazole biosynthetic gene cluster from marine-derived Streptomyces sanyensis FMA. Mar Drugs 11:466-488

Valentine BP, Bailey CR, Doherty A, Morris J, Elson SW, Baggaley KH, Nicholson NH (1993) Evidence that arginine is a later metabolic intermediate than ornithine in the biosynthesis of clavulanic acid by Streptomyces clavuligerus. J Chem Soc Chem Commun 1993:12101211

Wang L, Tian X, Wang J, Yang H, Fan K, Xu G, Yang K, Tan H (2009) Autoregulation of antibiotic biosynthesis by binding of the end product to an atypical response regulator. Proc Natl Acad Sci U S A 106:8617-8622

Ward JM, Hodgson JE (1993) The biosynthetic genes for clavulanic acid and cephamycin production occur as a 'super-cluster' in three Streptomyces. FEMS Microbiol Lett 110:239-242

Xu G, Wang J, Wang L, Tian X, Yang H, Fan K, Yang K, Tan H (2010) "Pseudo" gamma-butyrolactone receptors respond to antibiotic signals to coordinate antibiotic biosynthesis. J Biol Chem 285:27440-27448

Young TS, Walsh CT (2011) Identification of the thiazolyl peptide GE37468 gene cluster from Streptomyces ATCC 55365 and heterologous expression in Streptomyces lividans. Proc Natl Acad Sci USA 108(32):13053-13058

Zhou Z, Gu J, Du YL, Li YQ, Wang Y (2011) The -omics Era- a toward a systems-level understanding of Streptomyces. Current Genomics $12: 404-416$ 